Bio - grafía. Escritos sobre la Biología y su Enseñanza. ISSN 2027-1034

Edición Extraordinaria. p.p. 245 - 256

Memorias del IX Encuentro Nacional de Experiencias en Enseñanza de la Biología y la

Educación Ambiental. IV Congreso Nacional de Investigación en Enseñanza de la Biología.

\title{
EN DEFENSA DE LA MEMORIA Y EL TERRITORIO ANCESTRAL LAS MOYAS "CONTENEDOR DE VIDA"
}

\section{IN DEFENSE OF THE MEMORY AND THE ANCESTRAL TERRITORY FROM, OF MOYAS "LIFE CONT AINER"}

Karol J. Lozano $\mathbf{P}^{1}$

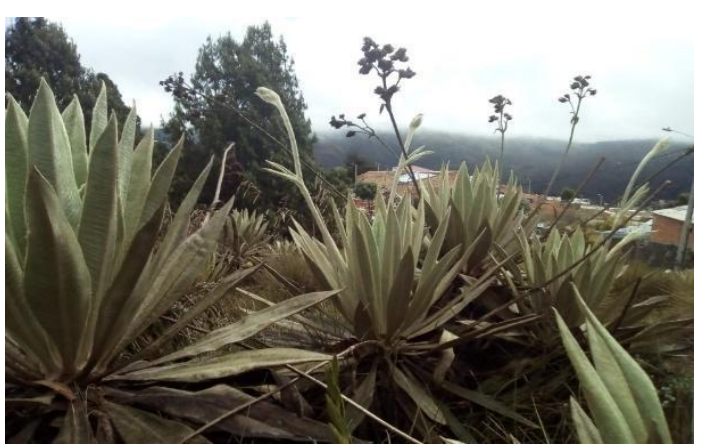

Si por el páramo no camina el agua, por las montañas no caminará la danta, por los mares no nadará la ballena, y el canto del Tángara se apagará así como la memoria de los pueblos.

\section{RESUMEN}

Este trabajo investigativo se enmarca en el proceso de formación de Licenciados en Biología de la Universidad Pedagógica Nacional, específicamente en práctica pedagógica I y II, dicho trabajo se realizó bajo el enfoque cualitativo, el cual permitió abordar integralmente el reconocimiento y categorización de los conflictos ambientales que se presentan en el territorio ancestral de las Moyas. Esto con el fin de contribuir a la resignificación del páramo las Moyas por parte de niños, niñas y mayores, quienes hacen parte de la comunidad y del acompañamiento que lleva realizando Casa Taller las Moyas hace más de doce años en el barrio San Luis ubicado en la ciudad de Bogotá.

Durante, este proceso investigativo fue posible reconocer y categorizar los conflictos ambientales según lo propuesto por (Palacio, 2001) en Tipo I hombre-hombre y Tipo II hombre - naturaleza, adicionalmente se incluye dentro de este reconocimiento la categoría de conflicto familiar, dicha categoría surge como reflexión del proceso de la practica pedagógica y del compartir con la comunidad de Casa Taller las Moyas.

\footnotetext{
${ }^{1}$ Karol Julieth Lozano Prada. Estudiante de Licenciatura en Biología dela Universidad Pedagogica Nacional
} 
Bio - grafía. Escritos sobre la Biología y su Enseñanza. ISSN 2027-1034

Edición Extraordinaria. p.p. 245 - 256

Memorias del IX Encuentro Nacional de Experiencias en Enseñanza de la Biología y la

Educación Ambiental. IV Congreso Nacional de Investigación en Enseñanza de la

Biología.

PALABRAS CLAVE: Práctica pedagógica, enseñanza de la biología, Casa Taller las Moyas, Territorio ancestral de las Moyas, Conflictos ambientales.

\section{SUMMARY}

This research work is framed in the process of formation of graduates in biology of the national Pedagogical University, specifically in pedagogical practice I and II, this work was carried out under the qualitative approach, which allowed to address Integral recognition and categorization of environmental conflicts that occur in the ancestral territory of the Moyas. This in order to contribute to the resignification of the moor the Moyas by children and adults, who are part of the community and the accompaniment that has been conducting workshop house the Moyas more than twelve years ago in the neighborhood of San Luis located in the city of Bogotá. During, this investigative process was possible to recognize and categorize environmental conflicts as proposed by (Palacio, 2001) in Type I man-man and type II man-nature, additionally included within this recognition the Category of family conflict, this category arises as reflection of the process of the pedagogical practice and of sharing with the community of House workshop the Moyas

KEY WORDS: Pedagogical practice, biology teaching, House workshop the Moyas, Moyas ancestral territorio, Environment conflicts.

\section{INTRODUCCIÓN}

Vivimos en una época de cambios profundos, de transformaciones políticas, sociales, culturales y ambientales que influyen en nuestra forma de concebir la complejidad de la vida, olvidamos aquello que nos ha hecho ser quienes somos, olvidamos de dónde venimos, olvidamos que somos vida que se expresa de diversas y maravillosas maneras, olvidamos a nuestros hermanos y todas las luchas que se han gestado por defender la vida en sus múltiples dimensiones. Esta es una de las razones para recordar que "en la memoria cada hora tiene su pájaro, cada minuto un pez que salta, un rugido de bestia invisible, un tambor escondido, el silbo de una flecha" (Ospina, 2008).

Pues, recordar, no es sólo hacer memoria de hechos o momentos que marcan el inicio o el final en las experiencias vividas, es rescatar un conocimiento que por generaciones se ha venido construyendo y transmitiendo, pero dicha memoria está siendo amenazada y destruida por las grandes industrias farmacéuticas, agroalimentarias, agroquímicas y mineras quienes buscan dominar, apropiarse y romper todo tipo de tejido social.

De esta situación no se escapa la Comunidad de Casa Taller las Moyas, pues quienes hacen parte de este proceso son seres que han sido o son víctimas de la violencia, el despojo, desempleo y de la desarticulación familiar, ante esta precaria situación surgen propuestas comunitarias que aportan a la defensa del territorio y por ende a la construcción de sujetos políticos, capaces de responder y transformar su realidad 


\section{Bio - grafía. Escritos sobre la Biología y su Enseñanza. ISSN 2027-1034}

\section{Edición Extraordinaria. p.p. 245 - 256}

Memorias del IX Encuentro Nacional de Experiencias en Enseñanza de la Biología y la

Educación Ambiental. IV Congreso Nacional de Investigación en Enseñanza de la

Biología.

inmediata, además de ser actores sociales con una elementos suficientes para decidir cómo vivir.

Casa Taller las Moyas-CTM- se encuentra ubicada en el barrio San Luis, UPZ 89, sobre los $3.200 \mathrm{msnm}$, en medio de un ecosistema muy frágil de subpáramo y bosque alto andino que actualmente se encuentra bastante fragmentado, este proyecto comunitario lleva doce años de resistencia y lucha por mantener el sueño de una familia que se reunió con unos intereses particulares; construir un espacio comunitario donde cada uno pudiera aportar desde lo que sabe, para esto se hicieron varias mingas, con el fin de construir un espacio para todos.

De este proceso hacen parte "[...] niños, niñas, jóvenes, adultos y mayores quienes nos hemos juntado para compartir, aprender y sobre todo des-aprender [....], basados en tres principios: autonomía, libertad, solidaridad, buscamos desarrollar el potencial humano, rescatando el saber popular y la posibilidad construir conocimiento de una manera colectiva aportando cada uno con lo que sabe".(Díaz, 2015).

Es así como Casa Taller las Moyas, resulta ser un espacio alterno a las instituciones educativas, pues allí, llegan jóvenes, niños y adultos, quienes reciben orientación y apoyo en sus tareas, por lo general se realizan talleres de panadería, tejido (crochet, macramé, chaquira), pintura, cerámica, agricultura urbana, elaboración de jabones y pomadas, talleres de yoga, música y teatro; esto con el fin de que se compartan conocimientos, y que lo jóvenes puedan aprender y participar de todas estas actividades que seguramente servirán para que cada uno tenga la posibilidad de elegir cómo vivir.

De esta manera Nicolasa dice: "un niño de Casa Taller entiende de una manera vivencial qué es ser solidario. Me sorprende es que, Casa taller es una casa física, pero esa dinámica ha trascendido y ya los de Casa Taller han aprendido a trabajar juntos en diferentes aspectos, como: recolección y manejo de la basura, la recuperación de quebradas, murales, etc. A través de esto, los niños empiezan a tener una apropiación por su territorio, a ser actores sociales activos capaces de transformar su realidad social" (Diario de campo, 2016).

Lo interesante de este proceso pedagógico comunitario, popular y para la vida, es que se parte de las problemáticas que se presentan en el territorio ancestral las Moyas, por esto, el trabajo de práctica pedagógica I, contextualizo el lugar, la comunidad, las prácticas culturales y las principales problemáticas presentes, a partir de lo encontrado, se plantea el objetivo de reconocer los conflictos ambientales locales a través de la activación de la memoria de la comunidad de Casa Taller las Moyas, esto con el fin de contribuir a la resignificación del páramo las Moyas "contenedor de vida".

Pues este páramo pertenece a los orobiomas alto andinos, ecosistemas que ofrecen importantes interacciones ecológicas como la capacidad de fijar el carbono atmosférico, retener y almacenar agua dando origen a las cuencas hídricas que nutren y abastecen casi el $70 \%$ la población colombiana (Rivera y Rodríguez, 2011). Su importancia no sólo es biológica y económica, sino que, dicha importancia ha sido reconocida por culturas precolombinas, quienes consideran el páramo como un lugar sagrado, base de una relación entre el hombre- tierra y hombre-montaña (González y Valencia 2011). 
Bio - grafía. Escritos sobre la Biología y su Enseñanza. ISSN 2027-1034

Edición Extraordinaria. p.p. 245 - 256

Memorias del IX Encuentro Nacional de Experiencias en Enseñanza de la Biología y la

Educación Ambiental. IV Congreso Nacional de Investigación en Enseñanza de la

Biología.

Ejemplo de ello es la comunidad de Casa Taller las Moyas, quienes han establecido una estrecha conexión con este espacio, como parte de la tramitación y actuación de los conflictos ambientales que se presentan en el territorio ancestral de las Moyas.

\section{METODOLOGÍA}

El trabajo investigativo de práctica pedagógica se enmarca en el paradigma cualitativo interpretativo. Desde este enfoque se utilizaron varias herramientas para la recolección de la información como: las entrevistas no estructuradas, conversaciones constantes, la escucha de las historias de vida, la observación participante, el diario de campo y grabaciones. Por otra parte se plantearon diversas actividades s para la indagación de los conflictos ambientales locales.

Cada actividad fue sistematizada de manera que se pudiera categorizar los conflictos ambientales encontrados, además de esto, el análisis de las entrevistas no estructuradas y las conversaciones permitieron saber, lo que reconocen por conflicto ambiental los jóvenes de CTM, encontrándose que el conflicto ambiental es una tensión diaria que toda la comunidad tiene que vivir, haciendo se explicito tensiones como: basuras, escombros, la contaminación de las quebradas y el páramo, el consumo de drogas y la pérdida de espacios para la recreación de los niños.

\section{Investigación sí, pero no así.}

En este apartado se resalta que, para la indagación, reconocimiento y categorización de los conflictos ambientales locales surgieron otras estrategias que generaron lazos de confianza, un diálogo de saberes y de historias de vida. Esto a su vez permitió la reflexión acerca del rol del Licenciada en Biología en estos contextos diversos y golpeados por la violencia epistémica y monetaria. Por esta razón se propone una metodología contextualizada que permita un acercamiento y establecer una relación de confianza y dialogo, y esta fue; compartir los espacios en los que niños y jóvenes se pueden expresar libremente, sin sentir presión por responder a lo que el investigador desea saber.

La mejor estrategia fue aprender, por esto desde cada espacio se iba escuchando y haciendo preguntas indirectas que permitiera el diálogo y ese encuentro entre todos, así que en este proceso de investigación pedagógica se propone una metodología desde el hacer, y este es el hacer de Casa Taller las Moyas:

\section{a) Caminatas por el territorio ancestral las Moyas "contenedor de vida"}

Sin lugar a duda, la única forma de reconocer el territorio como un espacio socialmente construido (Bozzano, 2013), es caminándolo y encontrándose como parte del él, en este sentido es curioso que a pesar de que la comunidad de Casa Taller hace parte la localidad de Chapinero, la gente que habita los barrios que se encuentran dentro de la UPZ 89, no se reconocen como parte de la ciudad de Bogotá. Por tanto su relación con el lugar donde habitan difiere mucho de la ciudad. 
Bio - grafía. Escritos sobre la Biología y su Enseñanza. ISSN 2027-1034

Edición Extraordinaria. p.p. 245 - 256

Memorias del IX Encuentro Nacional de Experiencias en Enseñanza de la Biología y la

Educación Ambiental. IV Congreso Nacional de Investigación en Enseñanza de la

Biología.

Esto se evidencio en las diferentes caminatas por el barrio, para llegar al Teatro CIAT se debe atravesar un bosque de chuscales y arbustos, para llegar al Parque la M se pasa por un relicto de páramo, para llegar a las Moyas se debe atravesar bosques propios de páramo, incluso para llegar a Casa Taller se debe trepar la montaña, todo esto hace que exista una relación ajena a lo que se vive o se entiende por ciudad.

Por esto, la importancia de caminar y reconocer actores, lugares, instituciones etc., del territorio donde se habita y se mantiene una relación muy estrecha, pues esto permite crear una cartografía de la memoria, de las dinámicas del territorio, y de las acciones que se toman frente a las tensiones que se viven.

\section{b) Cocinando al calor de la Tulpa}

Compartir en la cocina, fue una estrategia que permitió generar un acercamiento a los integrantes de la comunidad de Casa Taller, pues este espacio fue propicio para conversar, preguntar, escuchar las historias de vida, observar comportamientos, y frente a esto, indagar sobre los conflictos ambientales locales presentes en el territorio de las Moyas.

Por otra parte la Tulpa es un espacio educativo donde se aprende de una manera vivencial, se comparte saberes como las recetas para hacer pan, galletas, tortas, postres, pomadas, y el agüita de amor que todo el tiempo acompañó las tertulias y las onces preparadas por diferentes manos y compartidas con mucho amor para todos.

\section{c. Tejiendo sueños y utopías}

El tejido no es solo jugar con un hilo y la aguja formando diferentes nudos que te llevan a la construcción de algo. Tejer es establecer una relación intergeneracional, es escuchar, observar, es estar atento, pues a través del tejido se expresan sentimientos, pensamientos y experiencias, se expresa el ritmo circadiano y energético, por esto la importancia de retomar este tipo de prácticas donde se pueda expresar y compartir saberes que han sido transmitidos con mucho respeto y que mantienen un acervo cultural, esto permitió tejer puentes de comunicación entre el maestro investigador y el objeto de estudio.

\section{d. Minga por la chagra}

La minga como una estrategia de trabajo comunitario permitió reunir a los niños, jóvenes y mayores, en un mismo tiempo y espacio, compartiendo así, conocimientos acerca de las plantas, de las épocas de siembra y cosecha, de la elaboración de abonos, y de la identificación de semillas que se encuentran en Casa Taller las Moyas. Este encuentro tiene una gran significación en la vida de cada uno, pues cada quien asume una labor, responde por ello y lo comparte de manera vivencial con los demás, desde este espacio se problematiza el alimento, las plantas, el agua y la importancia de realizar acciones sustentables que permitan generar otras formas de relacionarse con el lugar en el que se habita. 
Bio - grafía. Escritos sobre la Biología y su Enseñanza. ISSN 2027-1034

\section{Edición Extraordinaria. p.p. 245 - 256}

Memorias del IX Encuentro Nacional de Experiencias en Enseñanza de la Biología y la

Educación Ambiental. IV Congreso Nacional de Investigación en Enseñanza de la

\section{RESULTADOS Y DISCUSIÓN}

Biología.

Durante todo el caminar por Casa Taller y el compartir sonrisas, alegrías, juegos, mingas, medicina, tejido y cocinar al calor de la tulpa, se pudieron reconocer algunos conflictos ambientales presentes en el territorio ancestral de las Moyas. Dichos conflictos surgen en el marco de la urbanización de sectores marginales por parte de familias víctimas del conflicto armado, despojo y la globalización.

\section{Reconocimiento de los conflictos ambientales locales por parte de la comunidad de Casa Taller las Moyas.}

En la fase de reconocimiento de los conflictos ambientales locales se identificaron varios, en esta parte se retomará los más relevantes, sin desconocer que todos están relacionados. Por tanto los conflictos ambientales explicitados a continuación, permiten aportar a la resignificación del páramo de las Moyas como un territorio de vida y saber.

\section{a. Reconociendo el páramo de las Moyas "contenedor de vida"}

Durante caminatas por el territorio las Moyas, se reconocieron algunas cuencas hídricas como la quebrada Moraci que nace el barrio la esperanza, y alimenta de agua a varias comunidades de los barrios aledaños. En este recorrido se buscó que los niños, niñas y jóvenes, observaran el estado y reconocieran las principales problemáticas, así se fue identificando y conversando, encontrándose gran cantidad de desechos como: ropa, bolsas plásticas, botellas, cajas etc., esto, es arrojado a la quebrada, o al bosque cercano, generando así, contaminación y alteración de este afluente hídrico.

Frente a este conflicto ambiental que ha sido reconocido por la mayor parte de la comunidad, Casa Taller las Moyas se ha vinculado activamente, participando de actividades como la recuperación de las quebradas Morací, La Sureña y Capilla, a este proceso se ha sumado la mayor parte de la comunidad, incluso hasta instituciones educativas, colectivos de jóvenes, el acueducto comunitario (Acualcos) y las mesas ambientales de este sector.

Casa Taller la Moyas en particular, ha aportado desde la marcación de senderos, recolección de basuras, siembra de árboles nativos, conversatorios, ollas comunitarias, compartiendo e involucrando a la comunidad en este proceso que implica generar concientización del lugar donde se habita, como se está habitando y el que hacer para mantener un equilibro.

En cuanto al páramo de las Moyas, se resalta que ha existido poca intervención por parte de la comunidad, pues en las caminatas se encontró gran cantidad de basuras, en los pajonales y frailejonales al igual que escombros en la zona arbustiva, a pesar de todo el esfuerzo de la comunidad por recuperar y apropiarse de su territorio, son complejas las dinámicas de vida que lleva la gente que habita este sector, a excepción de Casa Taller, quienes han intervenido con otro

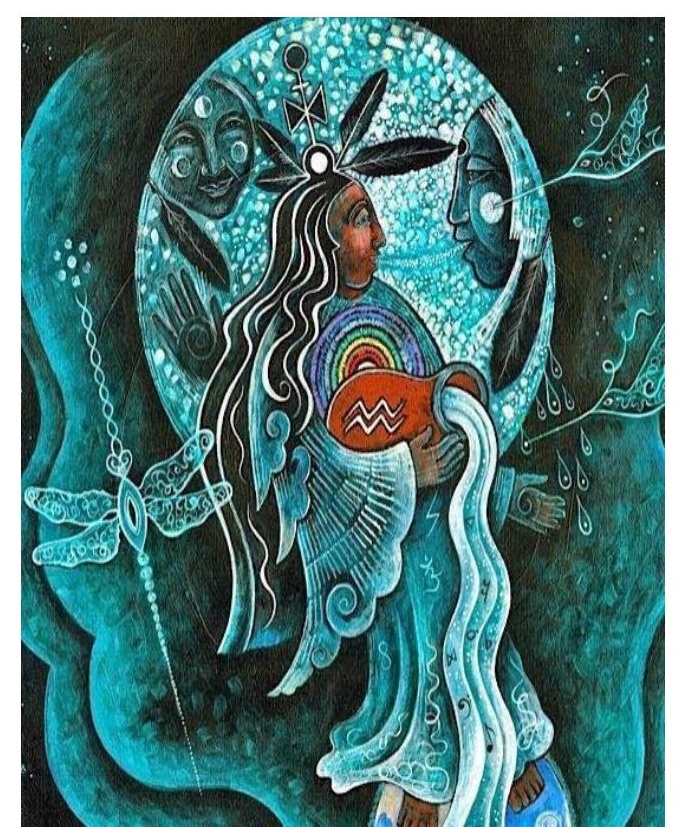


Bio - grafía. Escritos sobre la Biología y su Enseñanza. ISSN 2027-1034

Edición Extraordinaria. p.p. 245 - 256

Memorias del IX Encuentro Nacional de Experiencias en Enseñanza de la Biología y la

Educación Ambiental. IV Congreso Nacional de Investigación en Enseñanza de la

Biología.

tipo de relaciones; dichas relaciones son las prácticas ancestrales y espirituales, siendo esta una de las formas en las que se activa la memoria biocultural y se dialogan los saberes locales.

Dichas actividades consisten en realizar pagamentos, ofrendas y hacer cantos en las Moyas "Contenedor de vida" para los Muiscas, por tanto estas se realizan para reconocer el páramo como territorio de vida y saberes, buscando generar una transformación desde la conciencia y del comprender la integralidad del páramo con la gente y lo demás ecosistemas.

Es por esto que los niños, jóvenes y adultos, reconocen las Moyas, como un lugar sagrado de respeto y donde nace el agua, por esto al entrar se debe pedir permiso, se debe llevar una ofrenda y protección, se debe cantar agradeciendo a la madre por la vida, el agua y las plantas. En una de las salidas (Nicolasa Díaz, mujeres y maestra de CTM) enseña y comparte a todos el siguiente canto:

Aho, Aho, Aho gran espíritu

Aho, Aho gran espíritu

Del corazón de la montaña

Hacia el valle infinito

Del corazón de la montaña

Hacia el valle infinito

Aja, Aja, Aja, mi madre tierra

Aja, Aja, Aja, mi madre tierra

Madre de todas las criaturas

Todo lo que habita en ella

Madre de todas las criaturas

Todo lo que habita en ella

Rayo, Rayo, Rayo de un nuevo día

Rayo, Rayo, Rayo de un nuevo día

Late el tambor,

Danza del sol

Mi corazón siente alegría

Late el tambor,

Danza del sol

Mi corazón siente alegría 
Bio - grafía. Escritos sobre la Biología y su Enseñanza. ISSN 2027-1034

Edición Extraordinaria. p.p. 245 - 256

Memorias del IX Encuentro Nacional de Experiencias en Enseñanza de la Biología y la

Educación Ambiental. IV Congreso Nacional de Investigación en Enseñanza de la

Biología.

Aho, Aho.

(Se repite 4 veces, música de medicina)

Este canto nahuatl de culturas mesoamericanas, se hace para unir las energías, esto quiere decir que nos unimos para enlazar dos mundos. Por esto la importancia de que los niños generen otro tipo de relación más espiritual y sensitiva con un lugar como el páramo, esto ayuda a que sus relaciones interpersonales se dinamicen, se genere respeto por la vida y se aprenda escuchar y ser solidario (Diario de campo, 2016).

Esta experiencia fue posible evidenciar la mediante una actividad llamada en "busca del tesoro", para esta se utilizó la maleta didáctica de los Muiscas, la cual contiene piezas de gran valor cultural, esta maleta se llevó al páramo con la intención de que los niños, encontrarán dicho tesoro, y a partir de su sentir, oler, tocar, activar la memoria ancestral, viajar y recordar de dónde venimos, con esta actividad se evidenció el diálogo, el respeto por los mayores, por los saberes y la vida en general.

Dentro de esta actividad los niños y jóvenes de Casa Taller pudieron comprender, que los saberes no mueren, sino todo lo contrario, han sufrido algunos cambios, pero aún así, se mantienen. Fue de esta manera, como ellos reconocieron que Casa Taller mantiene prácticas ancestrales que les han sido heredadas como: tejer, trabajar la cerámica, sembrar y cultivar las semillas nativas de acuerdo al diagrama lunar, hacer los pagamentos agradeciendo todo lo que se aprende y se vive.

\section{b. Conflicto familiar: reconocido durante la investigación por la maestra en formación.}

El conflicto familiar fue reconocido por la maestra en formación durante la práctica pedagógica, este fue posible identificarlo, observando y escuchando, las formas en las que se relacionan los jóvenes, niños y niñas, a medida del compartir se fueron percibiendo ciertos comportamientos y lenguajes expresados en historias de vida, historias de la familia de los niños y niñas en edades que oscilan entre los 6 y 13 años.

Mediante diálogos y el convivir con niños y jóvenes, conocí sus conflictos familiares, presentándose en la mayoría: a) agresión física a las madres, b) descuido de los niños, c) drogadicción y alcoholismo, d) desintegración del tejido familiar.

Estos conflictos que fueron expresados por los niños, niñas y jóvenes, se presentan en sus hogares y están muy relacionados con el comportamiento que ellos tienen en otros escenarios y con otros sujetos. Por esto la importancia de complejizar este conflicto a nivel educativo, ya que muchos comportamientos que tienen la mayoría de estudiantes, son el reflejo de las relaciones familiares y por ende de un buen vivir de los niños.

Por otra parte, el rompimiento o desintegración del tejido familiar tiene una carga histórica, que está siendo evidente desde hace mucho tiempo, la cual se ha encargado de desarticular la familia, de romper el vínculo materno, paterno y de hermandad. Es así que se escucharon historias como: 
Bio - grafía. Escritos sobre la Biología y su Enseñanza. ISSN 2027-1034

Edición Extraordinaria. p.p. 245 - 256

Memorias del IX Encuentro Nacional de Experiencias en Enseñanza de la Biología y la

Educación Ambiental. IV Congreso Nacional de Investigación en Enseñanza de la

Biología.

“[...] mi papá murió cuando yo tenía 2 años, un día llegó borracho y no pudo abrir la puerta, él tenía las llaves en el bolsillo, pero de lo borracho se acordó, se metió debajo de la puerta y quedó atorado por el cuello con una lata, mis hermanos escucharon un ruido, pero como no teníamos luz no se supo quién era, ellos pensaron que era un ladrón y lo cogieron a pata, después de dieron cuenta que era mi papá". (Esneider, 8 años de edad, niño de CTM).

"Mi papa ahora es un jíbaro, la vez pasada me dijo téngame acá, yo cogí y era un saco, con una pistola, yo le dije no papá, y él me dijo Karen lléveme eso a la casa". (Diario de campo 2017).

La siguiente fue una conversación con tres niños que son vecinos y la maestra en formación:

- ¿Ya almorzaron?: No, contesta Esneider, a lo que Josep responde: la mama de Esneider se la pasa tomando y se gasta lo del mercado, llega borracha.

Preguntó a los otros niños ¿ustedes ya almorzaron?: No, mi mamá se fue desde esta mañana y no dejo nada.

En otra conversación con un niño de Casa Taller le preguntó: ¿hace cuánto que vives en este barrio?, él responde: "mi papá le pegó un puño a mi mamá en la cara y des de eso nos vinimos a vivir acá con mi mamá y mis abuelos" (Fabio, niño miembro de CTM)

Las anteriores conversaciones demuestran que existe un conflicto familiar de los cuales los niños terminan siendo las víctimas. Precisamente por esto nace Casa Taller las Moyas, pues se evidencia que existen fuertes y complejas relaciones sociales en las que conviven niños, niñas, jóvenes, adultos y mayores.

Con la creación de este espacio se busca unificar a la familia, desprender lo que se sabe y aprender a convivir, respetar, ser solidario, autónomo, sin agredir ni perjudicar al otro; por esto Casa Taller sigue incentivando el trabajo comunitario, a través de la huerta, la cerámica, el tejido, la tulpa, y la medicina como el Temazcal. La unión de estos elementos hace que los niños aprendan a convivir, compartir, escuchar y sentir que pueden transformar la realidad en la que viven desde su comportamiento, es así como tenemos el caso de cuatro hermanos; en una entrevista dice Lorena, joven de CTM: María, Josep, Freddy y Karen han cambiado mucho desde que llegaron acá, se acuerda que ellos antes era todos groseros y a todos les pegaban, ahora ya ellos esperan para que todos comamos, ya no son tan agresivos con el resto y participan en todo. (Diario de campo, 2017).

En definitiva el actuar que se ha gestado en Casa Taller frente a este conflicto, permite aportar a la pedagogía del amor, pues nadie aprende a golpes y gritos, la pedagogía del amor consiste en dar un abrazo caluroso de bienvenida y despedida, consiste en ese acompañamiento del proceso, del descubrimiento y de compartir arte y música: danza, teatro, yoga, temazcal. Esto despierta otras formas de sentir en los niños, de compartir con su compañero y ser solidario, de romper con el escudo que se ponen para defenderse del otro. 
Bio - grafía. Escritos sobre la Biología y su Enseñanza. ISSN 2027-1034

Edición Extraordinaria. p.p. 245 - 256

Memorias del IX Encuentro Nacional de Experiencias en Enseñanza de la Biología y la

Educación Ambiental. IV Congreso Nacional de Investigación en Enseñanza de la

Biología.

Así mismo sucede con los padres de familia que se acercan a ser parte de este proceso y acompañar a sus hijos, terminan transformando la relación con sus hijos, permitiéndose la restauración del tejido familiar. Con esto quiero hacer referencia a una actividad programada el día de las madres.

Se convoca a las madres y sus hijos para llevar a cabo un temazcal el día de la madre, esto con el fin de limpiar el tejido familiar y la relación que se gesta desde el útero. Fue así como llegamos unos 25 al llamado de la medicina, en esta sesión muchos jóvenes expresaron el agradecimiento y el perdón a sus madres y de paso a Nicolasa Díaz por todos sus esfuerzos para seguir manteniendo el proyecto comunitario construido por todos y para todos.

\section{c) Categorización de los conflictos ambientales}

Durante las actividades realizadas para el reconocimiento de los conflictos ambientales, emergieron bastantes tensiones, estas se categorizaron según lo que plantea Palacio, 2001: como tipos de conflicto ambiental Tipo I y Tipo II, los cuales dependen de la relación que se teja, en la categoría Tipo I, se reconocen conflictos que implican la confrontación entre seres humanos por la naturaleza; en el conflicto Tipo II se reconocen los conflictos que se dan entre naturaleza vs cultura.

Dentro del conflicto Tipo I hombre- hombre, se ubica en consumo de drogas y el despojo que se dio en el parque la $\mathrm{M}$, debido a que este, ha sido una disputa entre la misma comunidad que ha generado una preocupación, rechazo e indiferencia ante esta fuerte situación que viven en su gran mayoría los jóvenes. Esto ha llevado a que se presente deserción estudiantil y otros tipos de conflictos personales y familiares que inciden en la forma de comunicarse y de relacionarse con el otro.

Para los conflictos reconocidos dentro del Tipo II, se categorizó la abundancia de perros en la zona, pues este conflicto se ha presentado porque la gente en muchas ocasiones abandona los perros en la calle, contribuyendo así al crecimiento poblacional, lo cual incide en la desaparición de fauna debido a la caza de especies silvestres de páramo.

En esta misma categoría se reconoce el conflicto ambiental del páramo urbanizado, pues esta, es una situación compleja que presentan las zonas marginales de la Ciudad de Bogotá y más sectores que proveen gran cantidad de servicios ecosistémicos uno de estos el agua.

Por último se categoriza dentro de este tipo de conflicto, el consumo de la Espeletia por parte de una larva aún no identificada, dicho conflicto puede estar asociado a los cambios en la temperatura que se han presentado en los últimos años, en varios páramos de Colombia, pues este aumento en la temperatura ha hecho que algunas especies de franjas altitudinales más bajas puedan habitar ecosistemas como el de páramo, aprovechando los recursos y la condiciones de vida que este ofrece.

El tercer conflicto ambiental que se evidencia en la tabla, es el de Tipo Familiar, este conflicto fue reconocido durante las actividades y el compartir con los niños, niñas y jóvenes, y no es contemplado dentro de los tipos de conflictos ambientales propuestos por Palacio, 2001; ya que su descripción no acoge las características que presenta este conflicto, por esta razón se crea bajo otra categoría. 


\section{Bio - grafía. Escritos sobre la Biología y su Enseñanza. ISSN 2027-1034}

\section{Edición Extraordinaria. p.p. 245 - 256}

Memorias del IX Encuentro Nacional de Experiencias en Enseñanza de la Biología y la Educación Ambiental. IV Congreso Nacional de Investigación en Enseñanza de la Biología.

El conflicto ambiental-familiar, suele ser muy común en la mayoría de las familias, así lo han expresado los niños durante el compartir en actividades en Casa Taller, cabe resaltar que esta división de la familia tiene una carga histórica, que es necesario revisarla y comprender los fenómenos actuales. Por otra parte cabe aclarar que el conflicto familiar es una disputa que se dan entre los mismos integrantes de la familia, ya sea por algún objeto particular, comida, indiferencias, malos tratos, etc. Esta disputa ha llevado a casos de violaciones de los derechos humanos tanto de niños como de mujeres y hombres.

\section{CONCLUSIONES}

Se logra reconocer los conflictos ambientales locales mediante la metodología contextualizada propuesta, pues desde la tulpa, el tejido, las caminatas y las mingas se pudo establecer lazos de confianza, para dialogar, compartir e indagar acerca de los conflictos ambientales locales que se presentan en el territorio, en este sentido es necesario reevaluar las metodologías que se utilizan en la investigación cualitativa, pues, como próximos maestros es pertinente proponer metodologías que partan de la identidad y del que hacer de cada comunidad con la que se desee abordar un trabajo investigativo.

Durante el reconocimiento de los conflictos ambientales locales presentes en el territorio ancestral las Moyas, se pudo evidenciar la actuación y tramitación que ha venido llevando Casa Taller frente a dichos conflictos, esto permitió dialogar sobre las acciones directas que se han realizado para defender el territorio, además dio lugar para debatir sobre el rol que cumple Casa Taller las Moyas como un espacio de formación de niños, niñas, adultos y mayores. Esto a su vez, da a comprender que la crisis en la educación y la fracturación del tejido social, son mecanismos que limitan a que la escuela sea una comunidad empoderada que problematice de una manera contextualizada lo que acontece en los territorios.

En todo el compartir con la comunidad de Casa Taller las Moyas, se desdibujó la imagen del profesor o practicante, no fui reconocida como la profesora, sucedió todo lo contrario, me reconocieron como un miembro más de esta familia, en este sentido esta transformación reconfigura al maestro, como un sujeto colectivo e interlocutor entre los conflictos, capaz de repensar y reflexionar sobre su práctica pedagógica en contextos diversos, esto incluye el reconocer que existen otras formas de aprender a vivir, de relacionarnos con lo vivo y de construir nuestro propio saber pedagógico. A esto me ha llevado el compartir un año de sonrisas, comida, mingas, siembras, caminatas, bailes, música, palabra en Casa Taller las Moyas, pues este hermoso espacio me revive el compromiso con un cambio social, ambiental y cultural que posibilita la vida, las alegrías y las utopías, que aún no mueren, y que siguen vivas en cada uno de nosotros.

A lo largo de la investigación se logra evidenciar que los conflictos familiares inciden en la actuación que tienen los niños y jóvenes frente a los conflictos ambientales que se presentan en el territorio ancestral las Moyas, es decir, existe un vínculo entre los conflictos familiares y la degradación de los ecosistemas, pues si no aprendemos a amar y respetar a los seres con quien compartimos a diario, menos vamos a respetar lo que a nuestro alrededor se encuentra. Por este motivo hay que resaltar que Casa Taller las Moyas tiene una estrategia pedagógica para aportar a la solución de estos conflictos, y es 
Bio - grafía. Escritos sobre la Biología y su Enseñanza. ISSN 2027-1034

Edición Extraordinaria. p.p. 245 - 256

Memorias del IX Encuentro Nacional de Experiencias en Enseñanza de la Biología y la

Educación Ambiental. IV Congreso Nacional de Investigación en Enseñanza de la

Biología.

a través del reconocimiento del páramo las Moyas como lugar sagrado, esta estrategia ha permitido reconstruir el tejido social y poner en práctica la pedagogía del amor, de esta manera los niños y jóvenes de CTM se van transformando en sujetos capaces de comprender la integralidad del páramo, de generar relaciones de respeto con los otros, con las plantas, animales, el agua etc. Ahora son ellos quienes se preparan para defender y actuar frente a los múltiples conflictos que en su territorio se presenten.

\section{BIBLIOGRAFÍA}

- Bozzano, H. (2013). Territorios: el método territori. Una mirada territorial a proyectos e investigaciones no siempre territoriales. CONICET - UNLP. [En Línea]. Tomado https://halshs.archives-ouvertes.fr/halshs-00533337/document

- Díaz, N. (2015). (mujer, maestra y líder de Casa Taller las Moyas).

- Diario de Campo (2016- 2017)

- Gonzales, F.; Valencia, J. (2011). Los páramos en la historia. Revista Colombia tiene Paramos. Vol., Núm. 1. P. 4-21.

- Ospina, W. (2008). El país de la Canela. Monte Avila Editores, Caracas.

- Palacio (2001) Naturaleza en disputa. Ensayos de Historia Ambiental de Colombia 1850-1995. Universidad Nacional de Colombia. Facultad de Derecho, Ciencias políticas y sociales. [En Línea]. Tomado de http://www.bdigital.unal.edu.co/46808/6/9587010760.preliminares.pdf

- Rivera, D. y Rodríguez, C. (2011). Guía divulgativa de criterios para la delimitación de paramos de Colombia. Instituto de investigación de Recursos Biológicos Alexander Von Humboldt. 